\title{
Mechanisms of Strain Localization, Crack Initiation and Fracture of Polycrystalline Copper in the VHCF-Regime
}

\author{
Stefanie E. Stanzl-Tschegg ${ }^{1 \mathrm{a}}$ and Bernd Schönbauer ${ }^{1 \mathrm{~b}}$ \\ ${ }^{1}$ Institute of Physics and Materials Science, BOKU, University of Natural Resources and \\ Applied Life Sciences, Peter-Jordan-Strasse 82, A-1190 Vienna, Austria \\ ${ }^{a}$ stefanie.tschegg@boku.ac.at, ${ }^{b}$ bernd.schoenbauer@boku.ac.at
}

Keywords: Very High Cycle Fatigue (VHCF), strain localization, slip bands, PSB thresholds, stage I cracks, endurance limit, fatigue limit, fatigue damage, fatigue failure, polycrystalline copper.

\begin{abstract}
Development of areas with strain localization is regarded as first step of fatigue damage and failure. At small load amplitudes only very few such areas are generated, but their number increases with number of cycles, until all grains are covered by them. Slip bands become persistent (PSBs) during high enough numbers of cycles. Formation of numerous persistent slip bands together with deep intrusions or small stage I (mode II) shear cracks, however, is not sufficient to form a long and propagating crack and thus specimen failure below a specified strain/stress amplitude. Specimen fracture requires an approximately $100 \%$ higher cyclic load than generating PSBs. The threshold value for PSB formation is lower than traditionally reported. Quantitative results on cyclic plastic strain measurements, TEM observations of the dislocation structure in the VHCF regime and determination of the "short" crack length by use of a Kitagawa diagram are reported. Its relevance for fracturing and endurance limit is discussed.
\end{abstract}

\section{Introduction}

Fatigue damage at very high numbers of cycles (VHCF) has obtained increased attention during the last years, after it has been detected that high-strength steels may fail below the conventional fatigue limit after very high numbers of cycles, like $10^{9}-10^{10}$ under special conditions. It is well known that single-phase ductile materials, like face centred cubic copper do not show a fatigue limit. For the VHCF failures in high-strength steels inclusions have been identified as internal crack initiation sites [1-3], whereas PSB intensification, leading to intrusions and micro-cracks are regarded as surface fatigue crack initiation places in single-phase ductile materials [4-6]. Their role for crack initiation in the VHCF regime has been discussed by Mughrabi [4], and since a few years [7] extensive investigations on the formation, extension and intensification of slip bands have been performed with the ultrasonic fatigue technique up to $\sim 2 \times 10^{11}$ cycles in order to study the role of PSBs for fatigue failure. Different testing techniques were applied to study the development of slip bands into persistent slip bands. In the following, new investigations and results on topics, which have been treated in former studies already, will be reported.

- PSBs are formed not only above, but also below a certain cyclic stress/strain value, which has been considered as "PSB threshold" until recently (based on experiments at conventional frequencies and predictions from the plateau stresses in the cyclic stress-strain curves of single crystals [8]). Later [9], however, it has been shown that PSB formation does not only depend on the applied stress/strain amplitude, but also the number of cycles. Thus a preliminary value being $\sim 25 \%$ lower than the "conventional"-PSB threshold has been detected after $\sim 2.7 \times 10^{8}$ cycles [10], which is named therefore "VHCF"-PSB threshold in this paper.

- The relevant cyclic plastic strains were measured with different techniques and are smaller than mostly reported. Reasons for this and new experimental results will be discussed in this study. 
- To identify the PSBs and other features of slip localization, the polishing technique, direct SEM, Replica/SEM, AFM and TEM [7,8,10,12] have been used, and new TEM results will be reported in the following.

- Though intrusions and small cracks are formed together with the PSBs, fracturing does not take place at cyclic strains/stresses near these "VHCF" and "conventional"-PSB thresholds, but at approximately $100 \%$, or respectively, $50 \%$ higher stress/strain values after $\sim 10^{10}$ cycles.

\section{Material and Experimental Procedure}

Testing material was electrolytic copper, of either cylindrical shape with a constant diameter of $5 \mathrm{~mm}$ or hourglass shape with a diameter of $3 \mathrm{~mm}$ in the centre (for S-N measurements) and, respectively, hourglass shape with $3 \mathrm{~mm}$ thick rectangular cross section (for crack growth measurements). All specimens were heat treated in the same way, i.e. at $750^{\circ} \mathrm{C}$ for 75 minutes in vacuum. The resulting mean grain size was approximately $60 \mu \mathrm{m}$. Before testing, the specimens were polished mechanically and electrolytically. The Young's modulus was determined dynamically at ultrasonic frequency as well as statically in a bending and in a tension-compression experiment including measurement of the hysteresis. For all subsequent calculations $\mathrm{E}=130 \mathrm{GPa}$ was used.

The fatigue loading experiments were performed with a closed-loop controlled ultrasonic equipment at a testing frequency of $19 \mathrm{kHz}$. The displacement amplitudes are measured with a vibration gage and are used for feed-back control. In addition, the total strain amplitudes are measured with small strain gages. The accuracy of strain amplitude measurement was approximately $2 \%$ in all measurements. This means that, for example, the statistical variation of the stress amplitudes was between 0.3 and $1 \mathrm{MPa}$. Ultrasound tests are displacement, i.e. total strain controlled. Stresses (or loads) cannot be measured directly, but are determined from the measured total strain amplitude $\Delta \varepsilon_{\text {tot }} / 2=\Delta \varepsilon_{\mathrm{el}} / 2+\Delta \varepsilon_{\mathrm{pl}} / 2$. Subtracting $\Delta \varepsilon_{\mathrm{pl}} / 2$ from $\Delta \varepsilon_{\mathrm{tot}} / 2$, the elastic part $\Delta \varepsilon_{\mathrm{el}} / 2$ is determined. The axial stress amplitude $\Delta \sigma / 2$ is calculated using Hooke's law: $\Delta \sigma / 2=\mathrm{E} \cdot \Delta \varepsilon_{\mathrm{el}} / 2$.

The plastic strain amplitudes were determined indirectly by measuring the damping heat with micro-thermocouples in the ultrasonic tests [9]. For these measurements, the temperature rise during one pulse consisting of 20000 cycles or less, e.g. 500 cycles at higher total strain amplitudes $\left(\Delta \varepsilon_{\text {tot }} / 2\right.$ above approx. $\left.5 \times 10^{-4}\right)$ was recorded. The accuracy of measurement was $0.01^{\circ} \mathrm{C}$ and the statistical error of the plastic strain amplitudes therefore a few percent. In addition, the dissipated energies were determined by measurement of the hysteresis loop with micro-strain gages and a storage oscilloscope [9]. The total strain amplitude $\Delta \varepsilon_{\text {tot }} / 2$ was measured in the centre of an hourglass shaped specimen, and the elastic strain amplitude $\Delta \varepsilon_{\mathrm{el}} / 2$ on the extension piece, which is a tubular hourglass shape piece of Ti6Al4V alloy screwed in between ultrasonic horn and specimen. It can be estimated that practically only elastic deformation of this rod takes place. Thus the plastic part of the cyclic strain $\Delta \varepsilon_{\mathrm{p}} / 2$ can be determined by subtracting the (normalized) elastic portion $\Delta \varepsilon_{\mathrm{el}} / 2$ from $\Delta \varepsilon_{\mathrm{tot}} / 2$. The accuracy of measurement is limited by the strain-gage resolution of approximately $2 \%$. With this, the statistical error is by a factor of ten larger than for the thermoelectric values and therefore in the range of the absolute values of the plastic strains. Exactly the same procedure was used to determine the plastic strain amplitudes in the servo-hydraulic experiments in this study, after first measurements of the machine load data (to obtain $\Delta \varepsilon_{\mathrm{el}} / 2$ ) led to changing and obviously too high $\Delta \varepsilon_{\mathrm{p}} / 2$ values [10].

To simulate plastic strain controlled experiments the vibration amplitude (and thus total strain amplitude $\Delta \varepsilon_{\text {tot }} / 2$ ) is increased stepwise. The step height of the axial total strain amplitude was approximately $\Delta \varepsilon_{\text {tot }} / 2=8 \times 10^{-6}$ (corresponding to a stress increment of approximately $1 \mathrm{MPa}$ ) and the number of cycles was $2 \times 10^{4}$ cycles. The resulting plastic strain amplitudes $\Delta \varepsilon_{\mathrm{p}} / 2$ increase exponentially with $\Delta \varepsilon_{\text {tot }} / 2$ and are in the range of $5 \times 10^{-7}$ to $5 \times 10^{-5}$, which is 0.7 to 5.9 percent of the total strain amplitudes $\Delta \varepsilon_{\text {tot }} / 2$ in the investigated regime $\left(\Delta \varepsilon_{\text {tot }} / 2\right.$ between approx. $7.5 \times 10^{-5}$ and 
$8.7 \times 10^{-4}$ ) [9]. After this, the tests were conducted with the same ramp loading procedure at several specified total strain amplitudes $\Delta \varepsilon_{\text {tot }} / 2$ partly up to $1.7 \times 10^{11}$ cycles. In order to remove the damping heat, the specimens were cooled with compressed air, and pulse-pause sequences were performed. The pulse lengths were $200 \mathrm{msec}$ and the periodic pauses, depending on the vibration amplitude, between 500 and $2500 \mathrm{msec}$ so that the specimen temperature did not exceed approximately $25^{\circ} \mathrm{C}$ in the experiments. Measurements of fatigue crack growth $(\Delta \mathrm{a} / \Delta \mathrm{N}$ vs. $\Delta \mathrm{K})$ curves were performed with flat hourglass-shaped specimens with a thickness of $3 \mathrm{~mm}$, a width of $15 \mathrm{~mm}$ and a single $\sim 1$ $\mathrm{mm}$ deep edge notch. Crack propagation was observed with a video camera with special optics allowing magnifications of the specimen surface of 250x on the screen.

Measurements at $20 \mathrm{~Hz}$ and $0.2 \mathrm{~Hz}$ were performed with a servo-hydraulic machine (MTS) under load control using identical specimen material, shape and heat treatment. The specimens were ramp loaded like in the ultrasound test, with identical load steps of $1 \mathrm{MPa}$, but with less $\left(5 \times 10^{3}\right.$ instead of $2 \times 10^{4}$ ) cycles per step, after it could be shown in ultrasonic experiments that both ramps delivered comparable fatigue data.

\section{Results}

Results of measurement of the cyclic plastic strains in $19 \mathrm{kHz}-\mathrm{ultrasound}$ as well as $20 \mathrm{~Hz}$ and 0.2 $\mathrm{Hz}$ servohydraulic tests with different testing methods (thermoelectric and strain gage measurements of hysteresis area) are shown in Fig. 1.

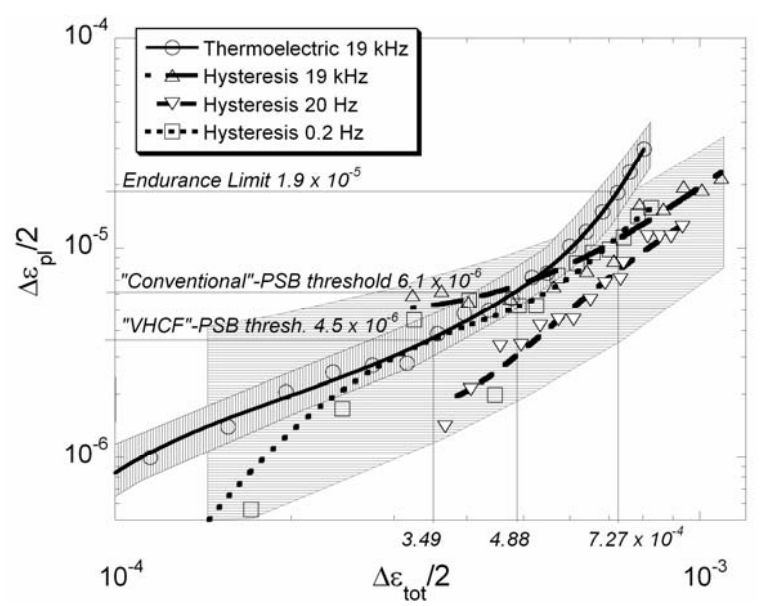

Fig. 1 Plastic strain amplitudes, obtained with different measuring methods at different total strain amplitudes at $19 \mathrm{kHz}, 20 \mathrm{~Hz}$ and $0.2 \mathrm{~Hz}$. Scatter ranges are indicated by shaded areas.

Indicated are also the scatter ranges, which are partly more than about ten times higher for the strain-gage than the thermoelectric measurements of the hysteresis loops, thus rendering rough estimates of the actual values only. Nevertheless, it is obvious that the difference between the results at the three frequencies is within the range of scatter. The slightly lower $\Delta \varepsilon_{\mathrm{pl}} / 2$ values at 20 $\mathrm{Hz}$ than at $19 \mathrm{kHz}$ are not statistically significant. A further reduction of the testing frequency to $0.2 \mathrm{~Hz}$ led to systematically slightly higher $\Delta \varepsilon_{\mathrm{p}} / 2$ at same $\Delta \varepsilon_{\text {tot }} / 2$ values and thus indicates the expected shift of the curves at a lower loading frequency. To summarize, Fig. 1 shows that an essentially similar $\Delta \varepsilon_{\mathrm{pl}} / 2$ versus $\Delta \varepsilon_{\text {tot }} / 2$ dependence is obtained for $19 \mathrm{kHz}, 20 \mathrm{~Hz}$ and $0.2 \mathrm{~Hz}$ measurements up to approximately $\Delta \varepsilon_{\text {tot }} / 2 \approx 7 \times 10^{-4}$, which means that a pronounced frequency effect does not exist. Above $7 \times 10^{-4}$, the thermoelectric values of $\Delta \varepsilon_{\mathrm{p} 1} / 2$ increase stronger; additional studies are necessary to explain this result. 
In addition, the values of the "conventional", "VHCF"-PSB thresholds and the endurance limit at $19 \mathrm{kHz}$ are introduced in Fig. $1: \Delta \varepsilon_{\mathrm{pl}} / 2 \approx 6.1 \times 10^{-6}$ for the "conventional"-PSB threshold (PSBs emerging within $2 \times 10^{6}$ cycles in analogy to literature measurements at conventional frequencies) and $\Delta \varepsilon_{\mathrm{pl}} / 2 \approx 2.9 \times 10^{-6}$ for the "VHCF"-PSB threshold (PSBs emerging in the VHCF regime) and $\Delta \varepsilon_{\mathrm{pl}} / 2 \approx 2.1 \times 10^{-5}$ for the endurance limit at $1 \times 10^{10}$ cycles. The indicated "VHCF"-PSB threshold is the lowest so far detected value for PSB formation (at $\Delta \varepsilon_{\mathrm{p} 1} / 2 \approx 2.9 \times 10^{-6}$ after $\approx 2.7 \times 10^{8}$ cycles) and is named "VHCF"-PSB threshold in the following owing to the very high number of cycles. Former investigations $[7,8,10,12]$ verified the persistence by using the polishing and reloading technique, SEM-replica and direct SEM as well as AFM observation. It is emphasized, however, that neither this PSB-threshold, nor the endurance limit are constant values, but depend on the number of cycles, being the lower the higher the number of cycles is $[9,10,12]$.

In order to further verify that slip bands, which are formed below the "conventional"-PSB limit, are indeed PSBs, TEM investigations were performed. TEM studies of a specimen, which was loaded at the ultrasonic frequency of $19 \mathrm{kHz}$ slightly above the "conventional"-PSB threshold $\left(\Delta \sigma / 2 \approx 64 \mathrm{MPa}, \Delta \varepsilon_{\mathrm{p}} / 2 \approx 6.1 \times 10^{-6} ; 1.5 \times 10^{10}\right.$ cycles) showed primary edge dislocations, many dislocation debris, elongated loop patches, cells and also a ladder-like dislocation structure [10,12]. These structures are known as typical for fatigue loading in the saturation regime and were also found at amplitudes close to the "VHCF"-PSB threshold and thus again verified that indeed PSBs have been formed.

At higher load amplitudes, similar cell structures have been observed, as shown, for example, in Fig. 2 for a stress amplitude $\Delta \sigma / 2 \approx 94 \mathrm{MPa}\left(\Delta \varepsilon_{\mathrm{pl}} / 2 \approx 2.5 \times 10^{-5}\right)$, after $\mathrm{N}=1.2 \times 10^{10}$ cycles, which is $1 \mathrm{MPa}$ above the endurance limit at $\sim 10^{10}$ cycles. One may recognize strongly elongated cells
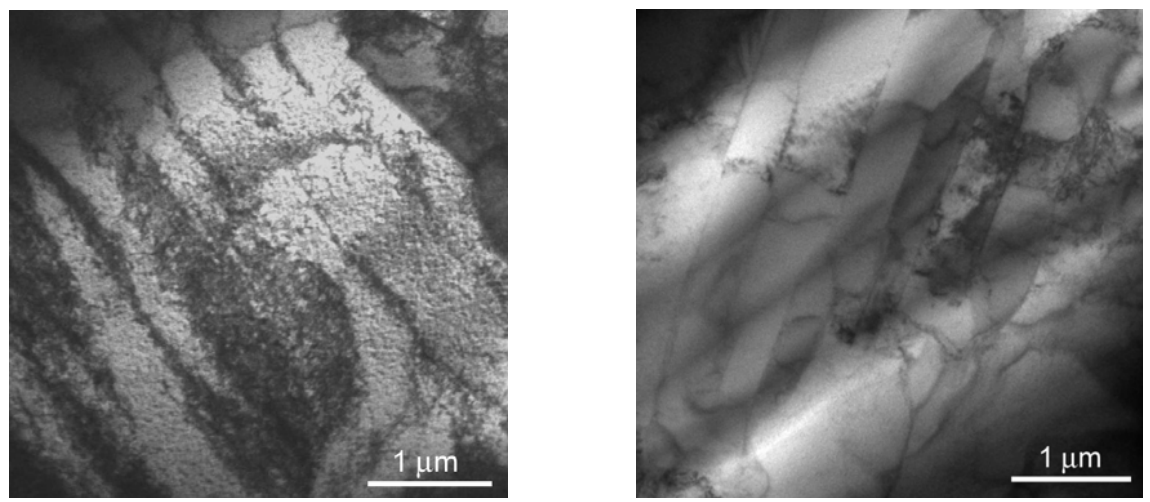

Fig. 2 TEM images of strongly elongated cells after $19 \mathrm{kHz}$ ultrasonic loading with $\Delta \sigma / 2 \approx 94 \mathrm{MPa}$ $\left(\Delta \varepsilon_{\mathrm{pl}} / 2 \approx 2.5 \times 10^{-5}\right)$ during $\mathrm{N}=1.2 \times 10^{10}$ cycles. a) cells and loop patches. b) very thin-walled cells.

(Fig. 2a) [10], which eventually are even more pronounced, i.e. show extremely thin cell walls and eventually look like small angle grain boundaries (Fig. 2b). These strongly elongated cells are similar to the cells and "lamellae" observed by Weidner et al. [11] by the FIB and ECC/SEM techniques in a specimen, which was fatigue loaded at $19 \mathrm{kHz}$ ultrasonic frequency in the same way (same ramp load procedure) at $\Delta \sigma / 2 \approx 61.5 \mathrm{MPa},(1.5 \mathrm{MPa}$ below the "conventional"-PSB threshold) in the specimen centre and which did not fail within $1.59 \times 10^{10}$ cycles. The elongated and thin-walled cells probably are the result of the extremely high number of cycles. Besides elongated cells, intrusions and microcracks have been detected (even at earlier stages of loading, like $10^{8}$ or $10^{9}$ cycles) $[7,8]$ and even in the specimen interior [11]. 
The stress/strain amplitudes, which lead to cyclic slip localization in form of PSBs and numerous small cracks are not sufficient to cause total fracturing, even at extremely high numbers of cycles like $1.7 \times 10^{11}[10]$. The "VHCF" as well as "conventional"-PSB thresholds may be considered as thresholds for the formation of PSBs and small cracks at specified numbers of cycles, but higher stresses are necessary to form a "long" crack. (For the difference of PSB and small crack formation see [13]). Measurement of the S-N and Coffin-Manson diagram revealed approximately $100 \%$ higher stress/strain amplitudes needed for failure [8,10,12] i.e. for developing (probably already formed) small cracks into one growing long crack. To further investigate this, fatigue crack-growth rate versus cyclic stress intensity $\left(\Delta \mathrm{a} / \Delta \mathrm{N}\right.$ vs. $\left.\Delta \mathrm{K}_{\mathrm{th}} / 2=\mathrm{K}_{\max }\right)$ curves have been measured (fracture mechanics tests) in this study, and the results are shown in Fig. 3a. A crack-growth threshold of $\sim 2 \times 10^{-14} \mathrm{~m} /$ cycle after $9.4 \times 10^{8}$ cycles was detected at a stress intensity threshold, $\Delta \mathrm{K}_{\mathrm{th}} / 2=\mathrm{K}_{\text {max,th }} \approx$ 3.4 MPa m${ }^{1 / 2}$ (Fig. 3a). Threshold stress intensities have been measured at different crack lengths (with different specimens). If the results are plotted in a Kitagawa diagram and correlated with the endurance limit $\Delta \sigma_{0} / 2$ of $93 \mathrm{MPa}$ (which was obtained after $\sim 1 \times 10^{10}$ cycles), a critical "short crack" length of $0.34 \mathrm{~mm}$ is derived (Fig. 3b).
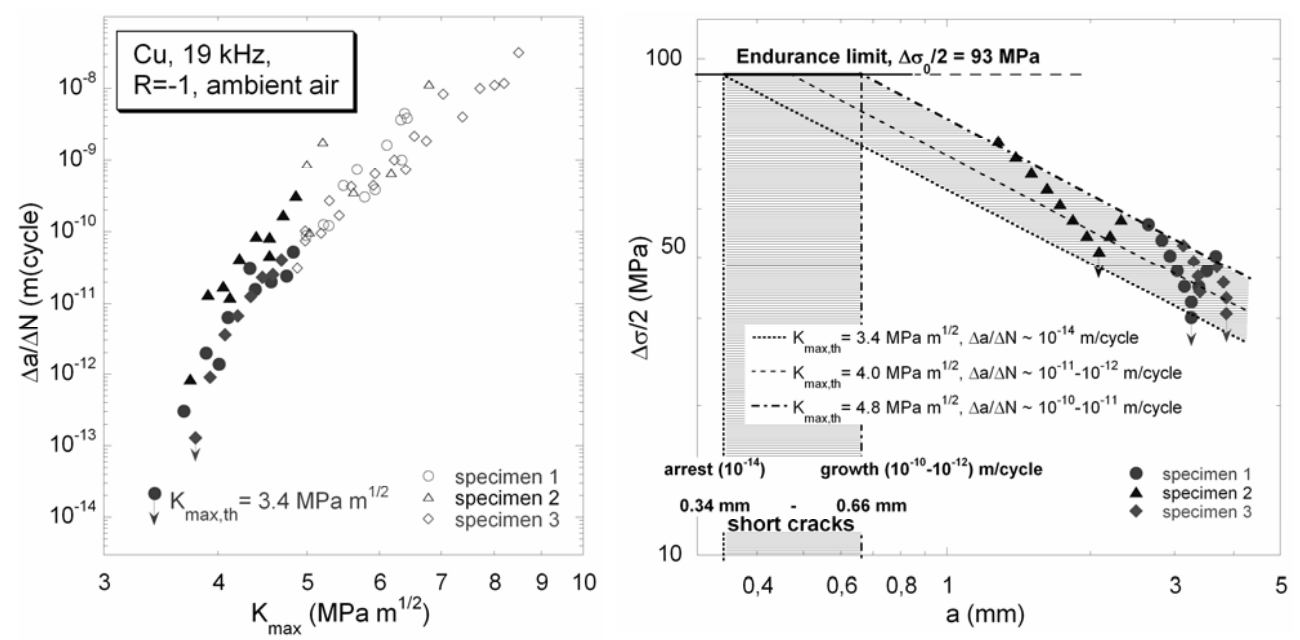

Fig. 3 Fracture mechanical determination of "long-crack" growth rates and "short"-crack lengths from

Kitagawa diagram. a) $\Delta \mathrm{a} / \Delta \mathrm{N}$ vs. $\Delta \mathrm{K} / 2=\mathrm{K}_{\max }$ curve, with a threshold $\Delta \mathrm{K}_{\mathrm{th}} / 2=\mathrm{K}_{\max , \mathrm{th}} \approx 3.4 \mathrm{MPa} \mathrm{m}^{1 / 2}$ at $2 \times 10^{-14} \mathrm{~m} /$ cycle. b) Kitagawa diagram, resulting in a short-crack length of $0.34 \mathrm{~mm}$ for $\mathrm{K}_{\max , \mathrm{th}}=3.4 \mathrm{MPa} \mathrm{m} \mathrm{m}^{1 / 2}$ and longer short-crack lengths (up to $\sim 0.66 \mathrm{~mm}$ ) for higher $\mathrm{K}_{\text {max,th }}$ values, derived from Fig. $3 \mathrm{a}$.

At slightly higher cyclic stress intensities $\left(\Delta \mathrm{K}_{\mathrm{th}} / 2\right.$ up to $\left.4.8 \mathrm{MPa} \mathrm{m}^{1 / 2}\right)$ very slow crack growth rates $\left(10^{-10}-10^{-13} \mathrm{~m} /\right.$ cycle) could be observed during $10^{7}-10^{8}$ cycles. Such low crack growth rates imply that the mean crack advance is far below one lattice spacing per cycle and usually not detectable in tests with conventional frequency so that they may be considered as thresholds. If also these higher $\Delta \mathrm{K}_{\mathrm{th}} / 2$ values of crack growth rates between $10^{-13}$ and $10^{-10} \mathrm{~m} / \mathrm{cycle}$ are evaluated in the Kitagawa diagram, longer "short"-cracks result. They are between 0.34 and $0.66 \mathrm{~mm}$ (Fig. 3b). 


\section{Discussion}

The following results will be discussed: Why are the plastic strain threshold amplitudes for PSB formation ("conventional" as well as "true" thresholds) lower at ultrasonic and conventional frequency than expected according to several literature results $[4,8]$ ? What is the influence of frequency? Why are considerably higher cyclic stresses (strains) necessary to cause failure, i.e. to form a propagating long crack than for introducing localized cyclic slip and small cracks?

The observed lower cyclic plastic strains may be explained by the different measuring techniques as used in this study and, respectively, in experiments with hydraulic machines usually. In this study, cyclic plastic strains were determined by thermoelectric measurement of the dissipated heat with micro-thermocouples and measurement of the hysteresis loops with microstrain gages $[7,10]$. Very low values of the cyclic plastic strain for "conventional" $\left(6.1 \times 10^{-6}\right)$ as well as "VHCF"-PSB threshold $\left(4.5 \times 10^{-6}\right)$ and the endurance limit at $1 \times 10^{10}$ cycles $\left(\Delta \varepsilon_{\mathrm{p} 1} / 2 \approx 1.9 \times 10^{-5}\right)$ have been detected in the experiments at $19 \mathrm{kHz}$ ultrasonic frequency [9]. Now, also measurements of the plastic strain amplitudes at conventional $(20 \mathrm{~Hz})$ frequency loading by detecting the hysteresis loop instead of using machine load data $\left(\Delta \varepsilon_{\mathrm{el}} / 2\right.$ from machine load) led to similar low values in the investigated $\Delta \varepsilon_{\text {tot }} / 2$ range between $6 \times 10^{-5}$ and $\sim 1 \times 10^{-3}$. The former used hydraulic machine data varied extremely (probably depending on electrical phase shifts of the machine signals) and therefore may explain the higher $\Delta \varepsilon_{\mathrm{pl}} / 2$ values reported earlier [9]. The former results of a "conventional"-PSB threshold at $\approx 57 \mathrm{MPa}$ at $20 \mathrm{~Hz}$ instead of $\approx 63 \mathrm{MPa}$ at $19 \mathrm{kHz}[9,10]$, however, still are valid.

Thus, the question about the reasons for the observed very low cyclic $\Delta \varepsilon_{\mathrm{pl}} / 2$ values $\left(\sim 3 \times 10^{-6}\right)$ for the onset of cyclic slip localization in the VHCF regime (at high as well as low frequencies) remains, after cyclic $\Delta \varepsilon_{\mathrm{p}} / 2$ values around $2.5 \times 10^{-5}$ were expected according to former studies on single crystals $[4,8]$. This value, however, is based on the assumption that the plastic deformation is homogeneous in the whole loaded volume. The above mentioned measuring techniques integrate the accumulated dissipated energy over a specified volume (and specified number of cycles), containing non-deformed grains besides grains showing plastic deformation. In the very low amplitude regime, only very few grains show plastic deformation [9] so that the integral measurement is mainly determined by non-deformed grains and thus renders lower mean values instead of the expected $2 \times 10^{-5}$. Lukáš and Kunz [3] have pointed to this effect and also mention that the degree of cyclic strain inhomogeneity is highest just in the VHCF region.

The 10-15\% higher cyclic stress/strain amplitudes of the "conventional"-PSB threshold at 19 $\mathrm{kHz}$ ultrasonic frequency $\left(\Delta \sigma / 2 \approx 63 \mathrm{MPa}\right.$ and $\left.\Delta \varepsilon_{\text {tot }} / 2 \approx 4.88 \times 10^{-4}\right)$ than at $20 \mathrm{~Hz}(\Delta \sigma / 2 \approx 57 \mathrm{MPa}$ and $\Delta \varepsilon_{\text {tot }} / 2 \approx 4.56 \times 10^{-4}$ ) [12] are probably caused by the higher strain rate leading to more pronounced hardening of the matrix material (frequency effect). But they may also result from the different ramp start procedure (with ten times higher numbers of cycles) and the ramping of each pulse in the ultrasound experiment, leading to a reduction of the localized strain in the PSBs [6,14], though no influence of a variation of the ramp load length on $\Delta \varepsilon_{\mathrm{pl}} / 2$ could be detected in the calibration experiments [7,9]. The value of $63 \mathrm{MPa}$ corresponds well with the shear plateau stress of $32 \mathrm{MPa}$ of Neumann for copper single crystals, which were also strongly ramp-loaded [13,14] and results of Yan and Laird [6] and Wang and Laird [15] on enhanced strain localization by ramp loading.

If it is tried to describe the mechanisms, which determine strain localization and fatigue failure of polycrystalline copper in the VHCF regime, the following results have to be considered:

- The "conventional"-PSB threshold $(\approx 63 \mathrm{MPa}$ at $19 \mathrm{kHz})$ is not a real threshold, since PSBs are formed at much lower cyclic strain/stress values, if the applied number of cycles is high enough. PSBs as well as small cracks are generated at cyclic stresses $\Delta \sigma / 2$ as low as $\approx 45 \mathrm{MPa}$ (lowest detected value until today with $\Delta \varepsilon_{\mathrm{p}} / 2 \approx 3.6 \times 10^{-6}$ ) after approximately $2.7 \times 10^{8}$ cycles [10]. Therefore this "VHCF" threshold may replace the "conventional" threshold. Number of PSBs 
and small cracks increase with the number of cycles [7,9], and it is expected that PSBs and small cracks are formed at even lower cyclic stress/strain values and higher numbers of cycles, since both, cyclic load and number of cycles determine their formation [12].

- Neither "VHCF" nor "conventional"-PSB threshold stresses/strains are sufficient to cause fracturing, even if more than $10^{11}$ cycles are applied and though full coverage of the specimen surface with PSBs and small cracks is obtained. Approximately two times higher cyclic stresses than for the "VHCF"-PSB threshold are needed to obtain failure.

From this the following failure mechanisms may be derived for the VHCF regime: At least above and probably also below (after high enough numbers of cycles) the "VHCF"-PSB threshold, irreversible deformation results in the formation of slip bands. With increasing numbers of cycles, the slip bands become "persistent" and give rise to the formation of intrusions, (preferentially at the borders of a slip band), which become deeper with time [7,9]. Voids are formed in the interior as well as in grain boundaries and close to the specimen surface and agglomerate preferentially in the direction of maximum shear stress and form small stage I shear cracks as detected in [11]. Similar elongated voids have been observed by Hunsche and Neumann, who used the taper sectioning technique and found "chains of large voids" along the trace of primary slip planes in copper single crystals [13]. Deep intrusions and, respectively, small stage I cracks especially at slip band borders, are formed in rather early stages of fatigue loading at each specified cyclic stress, as observed in SEM and AFM studies [7,9] and are assumed to be identical with those observed with FIB and ECC/SEM [11]. With increasing number of cycles, the number of PSBs and small cracks, i.e. features of strain localization, become more frequent and in addition more pronounced (deeper intrusions, longer and deeper small cracks) $[7,9]$. These processes take place in a volume, whose size depends on the material properties (modulus of elasticity, yield stress, etc.) besides the applied load. The volume, being strained/stressed above the "VHCF"-PSB threshold (which may be considered as micro-plasticity or elasticity limit or irreversibility limit) may be termed "plastic zone" or "process zone" similar to that of brittle materials, like concrete.

The numerous small cracks, however, obviously do not lead to failure below the "endurance limit" of $\Delta \sigma / 2 \approx 93 \mathrm{MPa}$, which is approximately two times higher than the "VHCF"-PSB threshold (and in the same range as the plateau stress, as observed by Wang and Laird on ramp-loaded polycrystalline copper [15]). This means that cyclic stresses below $\approx 93 \mathrm{MPa}$ and even very high numbers of cycles are not sufficient to form a growing "long" crack: For example, at a cyclic stress $\Delta \sigma / 2 \approx 92 \mathrm{MPa}$, the specimen did not fail within $1.7 \times 10^{11}$ cycles. More than $10^{20}$ cycles would be needed theoretically for fracturing according to an extrapolation of the SN curve.

Further information on the critical crack length, leading to a growing "long" crack and finally failure, may be obtained from the Kitagawa diagram. Measurements of this study revealed a "short" crack length regime below $340 \mu \mathrm{m}$. Indeed much shorter "small" cracks (max. $20 \mu \mathrm{m}$ ) have been observed by Weidner et al. [11] in a specimen, which was loaded slightly below the "conventional"PSB threshold. One must be aware, however, that a fracture mechanical measurement is not fully representative, as a single crack is induced there at the notch from the beginning in contrast to the very numerous "small" cracks, which are formed in fatigue loaded unnotched specimens. This means that the applied stress (intensity) is shared among (consumed by) numerous competing "small" cracks, so that the effective stress (intensity) is obviously too small to allow one crack (of a critical length of $340 \mu \mathrm{m}$, for example) to become a "long" crack, causing finally fracture. In addition, macroscopic stress relaxation of the loaded volume by the numerous small cracks may have led to a somewhat smaller effective stress. The observed much shorter "small" cracks anyhow make nonfailure at cyclic stresses in the PSB-threshold regime plausible.

In summary, these considerations may explain the big difference between "VHCF"-PSB threshold (threshold for cyclic slip localization) and endurance limit. Localization of strain is caused by PSBs and small crack formation, but fatigue failure by fracturing (and the endurance limit) is determined by the threshold stress necessary to propagate cracks to fracture. 


\section{Summary and Conclusions}

- A minimum stress/strain amplitude of $\Delta \sigma \approx 45 \mathrm{MPa} / \Delta \varepsilon_{\mathrm{p}} / 2 \approx 3 \times 10^{-6}$ has been detected so far, which is needed to form PSBs after $2.7 \times 10^{8}$ cycles and is named "VHCF"'-PSB threshold. But even lower threshold values are expected at higher numbers of cycles, since both, cyclic load and number of cycles determine the formation of PSBs. TEM studies show typical fatigue loading features in the range of the "VHCF"-PSB threshold with eventually strongly elongated cells.

- Lower plastic strain amplitudes than mostly reported have been determined with thermoelectric and micro-strain gage measurements in the very low amplitude range at ultrasonic as well as conventional frequencies $\left(\Delta \varepsilon_{\mathrm{p} 1} / 2 \approx 3 \times 10^{-6}\right.$ for the "VHCF"-PSB threshold and $\Delta \varepsilon_{\mathrm{p} 1} / 2 \approx 1.9 \times 10^{-5}$ for the endurance limit after $\sim 10^{10}$ cycles at $19 \mathrm{kHz}$ ). They probably represent mean $\Delta \varepsilon_{p l} / 2$ values of a few plastically deformed grains and a majority of non-deformed grains in the VHCF regime.

- Intrusions and "small" cracks are considered as early stages of fatigue damage. But even numerous small cracks do not cause failure by fracturing. Approximately $100 \%$ higher stress/strain amplitudes than for PSB and small crack formation are needed, which is obviously the crack driving force needed to form a propagating "long" crack. The length of the observed "small" cracks is approximately ten times shorter than that of "short" cracks, which have been determined by measurement of the cyclic threshold stress intensity $\left(\Delta \mathrm{K}_{0}\right)$ for "long" crack propagation and a Kitagawa diagram. This result may explain non-failure at amplitudes of the "VHCF"-PSB as well as "conventional" -PSB threshold.

\section{References}

[1] H. Emura and K. Asami, Trans. Jpn. Soc. Mech. Eng., Vol. 55A-509 (1989), p. 45.

[2] K. Shiozawa and L. Liu: Key Eng. Mat. 378-379 (2008) p. 65.

[3] Y. Murakami, T. Nomoto and T. Ueda: Fatigue Fract. Engng. Mater. Struct. 22 (1999), p. 581.

[4] H. Mughrabi: Fatigue Fract. Engng. Mater. Struct. 22 (1999), p. 633.

[5] P. Lukáš and L. Kunz, in: Proc. Int. Conf. on Fatigue in the Very High Cycle Regime, edited by S. Stanzl-Tschegg and H. Mayer, BOKU, Vienna, Austria (2001).

[6] B.D. Yan and C. Laird: Mat. Sci. Eng. 80 (1986), p. 59.

[7] S. Stanzl-Tschegg, H. Mughrabi and R. Schuller, in: Fracture of Nano- and Engineering Materials and Structures, Proc. ECF 16, no. 458, ed. E.E. Gdoutos, Springer (2006), ISBN-10 1-4020-4971-4.

[8] H. Mughrabi and R. Wang, in: Deformation of Polycrystals, Proc. $2^{\text {nd }}$ Riso Intern. Symp. on Metallurgy and Microstructures, ed. N. Hansen et al., Riso National Laboratory (1981), p. 87.

[9] S. Stanzl-Tschegg, H. Mughrabi and B. Schönbauer: Int. J. Fatigue 29 (2007) p. 2050.

[10] S. Stanzl-Tschegg, B. Schönbauer and C. Laird, in: Plasticity, Failure and Fatigue in Structural Materials - From Macro to Nano: H. Mughrabi Hon. Symp., ed. K.J. Hsia et al. TMS (2008), p. 229.

[11] A. Weidner, D. Amberger, F. Pyczak, B. Schönbauer, S. Stanzl-Tschegg and H. Mughrabi, in: This conference: $17^{\text {th }}$ European Conference on Fracture - Multilevel Approach to Fracture of Materials, Components and Structures (ECF17), edited by J. Pokluda and P. Lukáš, Brno, CR, (2008).

[12] S. Stanzl-Tschegg and B. Schönbauer, in: Proceedings of Fourth International Conference on Very High Cycle Fatigue (VHCF-4), edited by J.E. Allison et al., TMS (2007).

[13] A. Hunsche and P. Neumann, in: Basic Questions in Fatigue, Vol. I, ASTM STP 924, eds. J. Fong and R.J. Fields, ASTM, Philadelphia (1988), p. 26.

[14] B. Yan, A. Hunsche, P. Neumann and C. Laird: Mat. Sci. Eng. 79 (1986), p. 9.

[15] Z. Wang and C. Laird; Mat. Sci. Eng. 100 (1988), p. 57. 